\title{
Using Time-of-Day and Location-Based Mobility Profiles to Improve Scanning During Handovers
}

\author{
Paul Boone, Michel Barbeau and Evangelos Kranakis \\ School of Computer Science, Carleton University \\ Ottawa, Canada \\ \{pboone, barbeau, kranakis\}@scs.carleton.ca
}

\begin{abstract}
In WiMAX/IEEE 802.16 with mobility support, scanning for an available channel by a mobile station, especially during a handover, must be done promptly in order to reduce delays in network access. We have shown previously that mobile stations can reduce scanning times by maintaining a most probable list of frequencies in use. In this paper, we extend this idea to further capture the mobility patterns of users. By using time-of-day and location-based mobility profiles a mobile station improves scanning performance during handovers. We show this improvement by modeling and simulating an area of WiMAX coverage with various mobility patterns and real-world mobility traces.
\end{abstract}

\section{INTRODUCTION}

When a WiMAX/802.16 subscriber mobile station (MS) wishes to join the network, it must follow the network entry procedure. This involves scanning for a frequency on a base station (BS). It is expected that the MS be required to perform repeated scanning to maintain connectivity to the network by moving from one BS to another while moving throughout the coverage area. This process of changing BSs is called a handover.

Mobile WiMAX networks are expected to provide support for all Internet applications. High bandwidth and realtime applications, in a network with high mobility are still important open areas of research. WiMAX is considered as a competitive technology to replace or enhance existing 3G cellular networks. One of the most important issues that needs improvement in order to maintain its competitiveness is handover performance when dealing with large volumes of real-time data in a highly mobile environment [4].

In our previous work [6], we introduced frequency scanning strategies used to reduce the time a MS spends finding an available channel of a WiMAX/802.16 BS. These basic strategies are now implemented within user mobility profiles.

In this paper, we introduce time-of-day and location-based mobility profiles to aid MSs during the scanning phase when performing a handover. If a mobile user often follows the same set of routes, the MS can exploit this to its advantage. We would like to capture the fact that a user travels to work in the morning, returns home in the evening or heads out to the gym on Wednesday nights as either time-of-day or locationbased mobility profiles. Using these profiles, a MS reduces the number of frequencies monitored in the scanning process during handovers.

\section{A. Results of the paper}

We propose two mobility profiles, time-of-day (TOD) and location plus trajectory (LPT), used by MS in order to reduce the time spent searching for a frequency during handover scanning. We show a reduction in the time required for the scanning operation over our previous work [6]. Our strategies require no additional network support and only limited memory and computational resources of the MS.

We evaluated the use of TOD and LPT mobility patterns by modeling and simulating an area of WiMAX coverage with two MS user classes along with two different mobility models. In addition, we use real-world mobility trace data to evaluate the model.

The remainder of the paper is organized as follows. In Section II, we describe the WiMAX/802.16 network entry procedure and handovers. In Section III, we briefly revisit our previous work in order to give a background to our new mobility profiles. we also discuss other previous work. In Section IV-A, we introduce the TOD user mobility profiles. In Section IV-B, we introduce LPT user mobility profiles. We briefly analyze the Manhattan mobility pattern in Section V. We provide a description of the simulation environment along with the simulation results in Sections VI and VII. Finally, we discuss ongoing work and conclude in Section VIII.

\section{Network Entry AND Handovers IN WiMAX}

WiMAX/802.16 defines a network entry procedure for a MS wanting to establish a network connection via a BS. The MS must first scan to find a frequency in use by a BS. It does this by listening to each possible frequency until it hears the frame preamble. This takes a minimum of two frames [12], at each frequency. After finding the frequency, it must synchronize with the BS by waiting for the Downlink Map (DL_MAP). The DL_MAP is a map of the timeslot locations in use for the frame. Once the MS has synchronized with the channel, it then must listen for the Downlink and Uplink Channel Descriptors (DCD and UCD) that are periodically sent by the BS. Then the MS must wait for a contention slot (determined from the UCD) in order to perform Initial Ranging with the BS. Initial ranging is used to determine the transmit power level of the MS in order to reach the BS.

When executing a handover, the MS must perform the steps of network entry with a neighbor of the currently serving BS. Since the network entry process has many steps, if we can 
improve upon the time it takes for one or more of these steps to complete we can provide an improved access time of service. In this paper, we focus on handover scanning.

\section{A. Scanning in WiMAX}

Scanning is an activity conducted by a MS. The goal of scanning is to acquire a downlink signal from a BS. Scanning is done by monitoring each possible frequency until a downlink signal is received. The exact number of available frequencies depends on the regulatory provisioned bandwidth (varies from one country to another), physical specification (several) and bandwidth per channel (several options available per physical specification). Scanning is performed during the initial network entry procedure and continues periodically to aid the MS in the selection of a suitable target BS for a handover to maintain network connectivity while in motion.

WiMAX/802.16 provides optional support for network assisted handovers where the BS currently serving the MS can obtain the information of neighboring BSs over the network. The serving BS periodically sends this information as a MOB_NBR-ADV message to the MSs.

\section{B. WiMAX Handovers}

As a MS moves throughout a coverage area, maintaining connectivity is done via performing handovers between neighboring BSs. Selection of the best handover target can be complex since the MS must scan for neighboring BSs to find a suitable target based on a number of criteria such as signal strength or error rates. Since a handover is an important function, a MS should perform the scanning and determine a target BS before beginning the handover. The IEEE 802.16 standard supports temporarily suspending the uplink and downlink communication between a MS and a BS in order to allow the MS to perform scanning for neighboring BSs. While communication is suspended, the data streams must be buffered on either side. Any improvement on the time it takes for the MS to complete its scanning operation improves the performance of the communications, reducing delays.

While WiMAX supports three kinds of handovers: hard handover, Fast Base Station Switching (FBSS), and Macro Diversity Handover (MDHO), hard handover is the only mandatory scheme [5]. Since a hard handover is a break then make mechanism, data being sent across a connection is more sensitive to delays in connecting to the new BS. Additionally, according to WiMAX/802.16, handovers must be completed with a latency of less than $50 \mathrm{~ms}$ [1].

\section{REVisiting ScAnNing Strategies And PREvious WORK}

In this section, we briefly give a background of our previous work as well as other related work.

\section{A. Scanning Strategies}

When a MS is turned on for the first time, all frequencies are equally likely since the MS has no history. That is to say if there are $n$ frequencies, that the frequencies $f_{1}, f_{2}, \ldots, f_{n}$ have an initial probability distribution $p\left(f_{1}\right), p\left(f_{2}\right), \ldots, p\left(f_{n}\right)=\frac{1}{n}$.

From the initial setup, since a MS has no previous history, it must simply start scanning frequencies in increasing order. As the MS performs a number of successful scanning operations, it can determine an order of frequencies from its observations. This history can be used to make scanning more efficient.

We previously proposed two different kinds of strategies to aid a MS in frequency selection for scanning. The first was the most general. A MS built a profile based on its history of successful scanning operations. This was based only upon the frequencies the MS had previously used. The second strategy leverages the optional neighbor advertisements from a BS along with a profile built from the MSs history of handovers BS pairs.

\section{Frequency Scanning Strategy}

In our Most Frequently Used (MFU) strategy, a frequency of occurrence distribution over the radio frequencies is built using the history of the number of times each frequency is used. The frequency that is used the most has the highest probability. The MS keeps an ordered list starting with the most frequently used frequency. In future scanning operations, the MS scans the frequencies from the most frequently used to the least frequently used frequencies.

\section{Previous Handover Strategy}

In this strategy, we assume that the MS is provided with the list of all neighboring BSs and their frequencies in a MOB_NBR-ADV message from its serving BS. Even though this information is provided to the MS, the MS must guess at which of the neighboring BSs it should attempt to perform a handover. The MS builds, for each BS it visits, a most probable list of handover target neighbors based on its previous handover history. The MS uses this list, along with the MOB_NBR-ADV message, to aid in selecting future handover target BSs.

\section{B. Previous Work related to Scanning and Handovers}

The authors in [12] introduce their Adaptive Channel Scanning (ACS) algorithm. ACS is primarily focused on when to perform scanning by estimating the time required for a MS to scan a list of neighboring BSs and then interleaving the scanning and data transmission intervals. The authors in [5] propose an optimization of MAC layer management messages for networks that have relay stations. They introduce new management messages for coordination information requests and association and coordination information responses. Another early work describes the storing of information on the most probable used carrier frequencies in cellular networks on the MS [13]. However, the term most probable is not defined and no mechanism is provided for determining the most probable frequencies.

Other work has been focused more specifically on improving handover performance. The authors in [7] introduce a new management message to enable the reception of downlink data 
during the handover process and thus reduce the downlink packet delay. Their proposed algorithm allows a MS to receive downlink data from the handover target BS after synchronizing with the downlink channel, but before synchronizing with the uplink channel. The authors in [11] proposed Last Packet Marking (LPM) that requires integrating the MAC layer (L2) handover and network layer (L3) handover. Both handovers happen concurrently. Finally, the authors in [9] propose a cross-layer fast mobile IPv6/802.16e handover scheme (CLHS). They send FMIPv6 handover information with the mobile handover indication and range request WiMAX/802.16 messages.

\section{Using Mobility Profiles to Improve SCANNING}

We describe our extended scanning and handover strategies with the introduction of two mobility profiles, time-of-day (TOD) and location plus trajectory (LPT).

\section{A. Time-of-day Mobility Profile}

We introduce our time-of-day (TOD) mobility profile. The MSs uses time-of-day information when building and maintaining their user mobility profile. Things that can be taking into consideration are:

1) Time of the day (morning, evening etc.).

2) Day of the week (weekday vs. weekends).

3) The current user, for multiple users.

For each time-of-day period, the MS keeps a list of most probable frequencies used during the period.

When a MS is about to perform a scanning operation, it looks up its time-of-day profile to determine the order of frequencies to check. Figure 1 shows a sample lookup table for two time-of-day profiles $T O D_{1}$ and $T O D_{2}$. For $T O D_{1}$, frequency $f_{2}$ is selected first, then $f_{1}$ while for $T O D_{2}$ the reverse is true. $T O D_{(x, y)}$ represents time-of-day period $x, y^{t h}$ frequency.

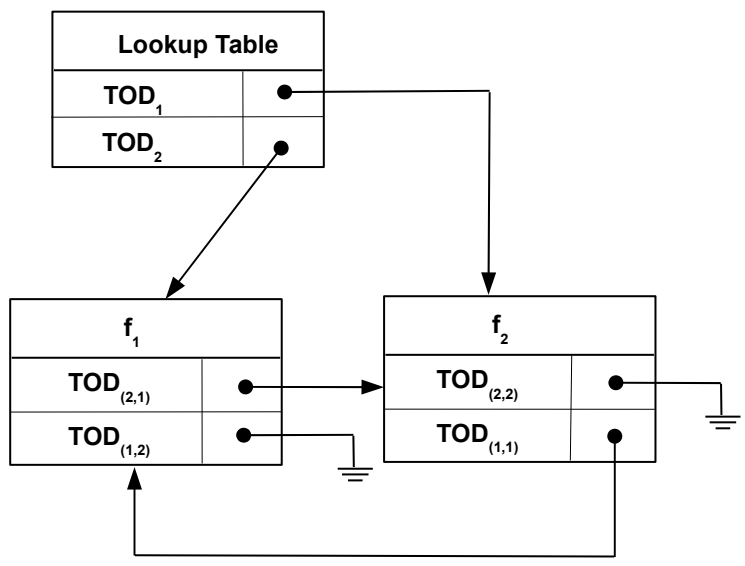

Fig. 1. Mobile station lookup for time-of-day mobility profile frequency ordering.

We assume that the first time a MS is started, the set of frequencies are of equal probability. Over time, the MS builds a set of mobility profiles. Each profile has its own ordering of the set of frequencies. The MS determines the number of mobility profiles to store based on its own timeof-day mobility patterns. The MS has a function that takes the time-of-day as a parameter and performs a table lookup for which time-of-day mobility profile to use. It then uses the most probable list of frequencies associated with this time-ofday profile.

\section{B. Location Plus Trajectory Mobility Profile}

We introduce our location plus trajectory (LPT) mobility profile. In the previous section, we presented a mobility profile that maintains lists of most probable frequencies depending upon the time of day or day of the week. This did not take into consideration information such as current location or direction of travel. In the LPT mobility profile, a MS equipped with a GPS or other means of localization uses this information when building its most probable frequency lists.

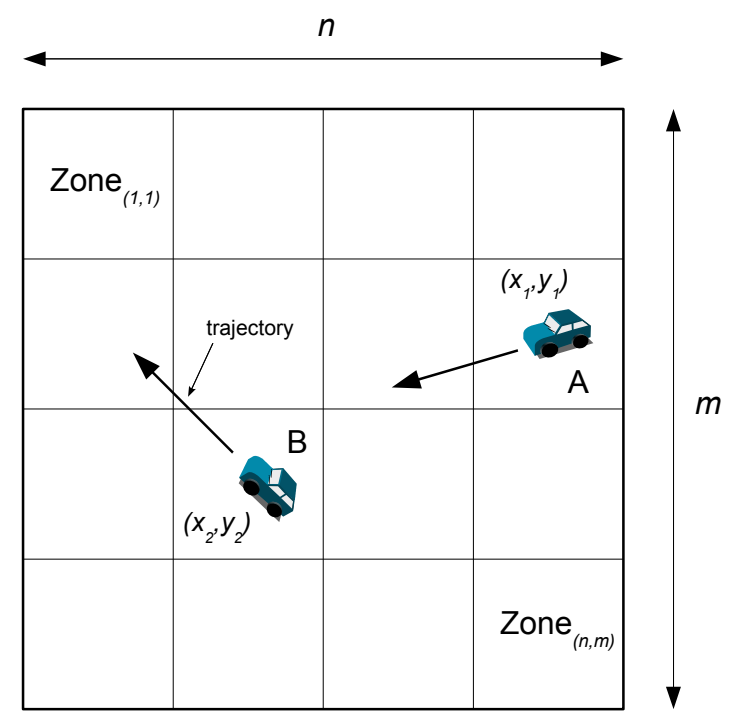

Fig. 2. Overview of location plus trajectory.

A MS uses its location as well as its trajectory to build a profile of several most probable frequency lists. The scene is depicted in Figure 2. We see MS A and MS B. Given an area of coverage, the region is divided into $n \times m$ zones. For each zone, the MS keeps a set of lists as a function of the trajectory of the MS within the zone.

As an example, Figure 3 shows how the MS determines which list from the current zone to use when performing scanning. In this example, the zone has four related lists, one for each quadrant Q1 through Q4. The MS determines which list to use by calculating which quadrant it is located in by using its current and previous locations. A lookup is performed in a similar fashion as the TOD mobility profiles shown in Figure 1, with the exception that each zone has multiple most probable lists as compared to the single TOD most probable list. 


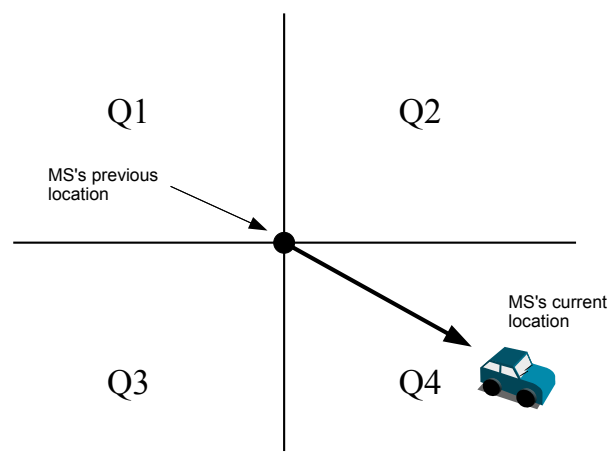

Fig. 3. Determining a mobile station's trajectory.

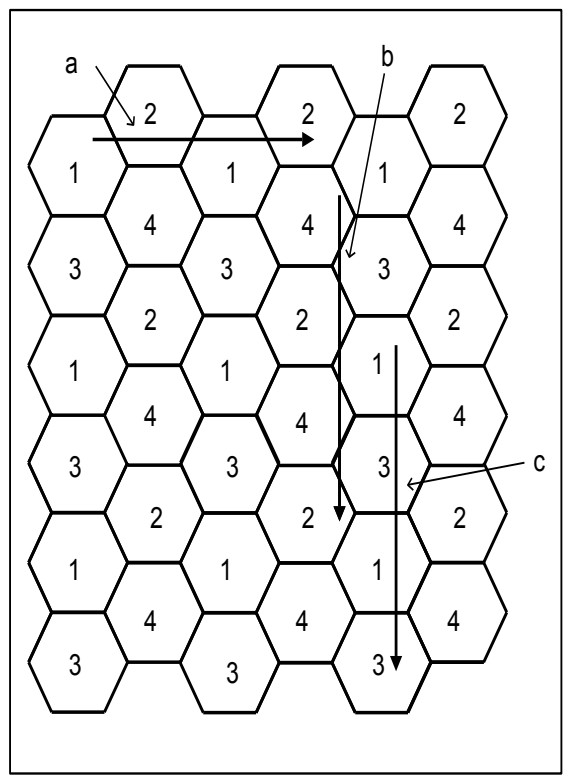

Fig. 4. WiMAX coverage, frequency use, four cell clusters, Manhattan mobility model.

\section{Analysis of Manhattan Mobility Model}

To give some intuition on why maintaining a most probable list of frequencies to check during scanning as mobility profiles improves scanning time, we investigate the properties of a MS's path in the Manhattan model [8].

Refer to Figure 4 where we show a sample of three segments of possible MS paths and how the MS visits a set of BSs in the horizonal and vertical directions in the Manhattan model with a four cell clustering scheme. Cells labeled 1 through 4 are each assigned $\frac{1}{4}$ of the available frequencies. In the case of horizontal movement, the segment indicated by "a" in Figure 4 , a MS visits a set of BSs that at most contain $\frac{1}{2}$ the total number of frequencies (BSs that fall in to cells numbered 1 and 2 of the four cell cluster).

In the vertical movement case, we see two cases of the set of BSs visited by a MS. The first is similar to the horizontal case and is depicted in Figure 4 the segment indicated by "c". The latter is shown in Figure 4, the segment indicated by "b", where the MS may traverse a set of BSs that include all the frequencies in use.

Examine Figure 5 where we depict the area of a BS cell. We can calculate the area of the three sections and determine the probability of a user traveling, in the vertical direction, through each part. Given a unit hexagon, the area of the triangle on the left hand side, with a base of $\sqrt{3}$ and a height of $\frac{1}{2}$ is $\frac{1}{4} \sqrt{3}$ units $^{2}$. We multiply this by 2 in order to get the area of both triangles, giving us $\frac{1}{2} \sqrt{3}$. The area of the central part is $\sqrt{3}$ units $^{2}$. The triangles represent $\frac{1}{3}$ of the area and the central part represents $\frac{2}{3}$ of the area of the hexagon.

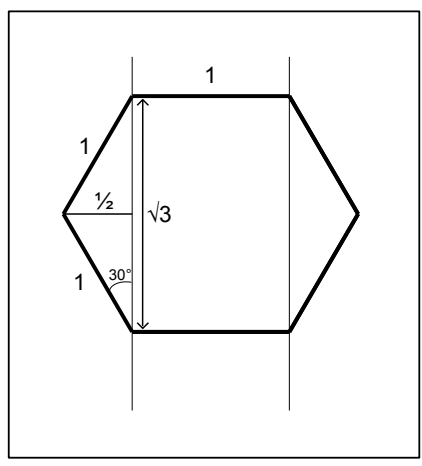

Fig. 5. Calculating the area of a cell.

When moving in the vertical direction, the proportion of time a MS may use BSs that cover all of the available frequencies is only $\frac{1}{3}$ of the time (as with segment " $b$ " in Figure 4) versus $\frac{2}{3}$ of the time when it only uses BSs that cover $\frac{1}{2}$ of the available frequencies (as with segment "c" in Figure 4).

In the Manhattan mobility model, this leads to the conclusion that with a higher probability, a MS repeatedly following similar paths is only exposed to $\frac{3}{4}$ of the frequencies in use in the area of coverage (assuming fixed frequency assignments). If a MS can check these frequencies first while scanning, it should gain an advantage.

\section{PERformance Evaluation}

In order to evaluate the model, we first implemented a simulation to compare the WiMAX/802.16 default scanning strategy with the original MFU strategy and that of the TOD and LPT mobility profiles. Similarly, we implemented a second simulation to compare the WiMAX default strategy, our original previous handover strategy and the TOD mobility profile for the previous handover strategy.

We first describe important parameters concerning the simulation: Network topology, metrics and measurements being used, the Mobile Station mobility patterns used, and time-ofday during the weekdays. Later we discuss the results of the simulations.

\section{A. Network Topology}

A ten kilometres by ten kilometres area is defined and covered by WiMAX/802.16 BSs with a one kilometre range. The 
base stations are positioned according to a cellular networking model [3] where each BS covers a hexagonal shaped region as shown in Figure 6. A total of 44 BS are used to cover the entire area.

\begin{tabular}{|c|c|}
\hline \multicolumn{2}{|c|}{ Network Parameters } \\
\hline Coverage Area & $10 \mathrm{~km} \mathrm{x} 10 \mathrm{~km}$ \\
\hline No. Base Stations & 44 \\
\hline Cell Radius & $1 \mathrm{~km}$ \\
\hline Cell Cluster Size & 4 \\
\hline No. Frequencies & 20 \\
\hline No. Channels & 80 \\
\hline No. Channels per Cell & 20 \\
\hline No. Frequencies per cell & 5 \\
\hline TOD Periods & Weekday AM, Weekday PM \\
\hline LPT Zones & 16,25 \\
\hline LPT Trajectory Lists & Quadrant based \\
\hline
\end{tabular}

TABLE I

SIMULATION PARAMETERS.

The WiMAX/802.16 simulation parameters are chosen based on $100 \mathrm{MHz}$ of bandwidth available for both uplink and downlink channels. Every channel is given $1.25 \mathrm{MHz}$ of bandwidth for a total of 80 channels. A four cell reuse clustering scheme is implemented on the underlying 44 BSs. This setup has 20 channels available for each cell for the 80 channels available in total. We use 20 different frequencies, assigning five to each BS, each of which has four channels for MS connections. In the model, the number of frequencies stored in memory is equal to the number of frequencies available in the coverage area. Each simulation using the Manhattan and Direct mobility models was run for a period of 100 weekday work days for 100 mobile stations. The simulation network parameters are summarized in Table I.

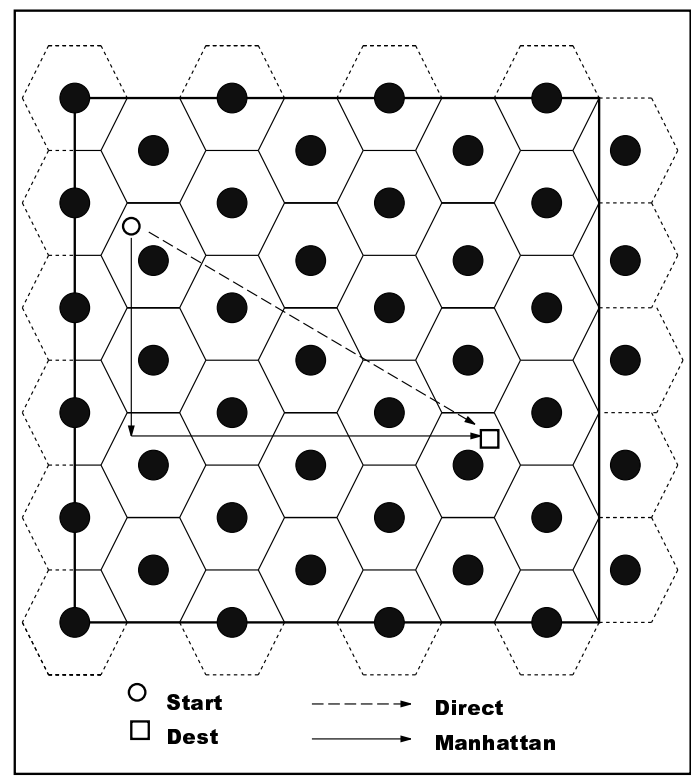

Fig. 6. Simulation area with sample paths of a mobile station.

\section{B. Metrics and Measurement}

The purpose of this work is to improve upon (reduce) the number of frequencies a MS is required to check while scanning for a downlink connection to a BS. We measure the proportion of time that a MS can find the downlink with a certain number of frequencies checked. For the model and the frequency strategies, it is between one and 20 frequencies checked per scan. For the previous handover strategy, it is between one and six neighboring BSs. Ideally, we would like to have it so that $100 \%$ of the time a MS can successfully scan for a neighboring BS with only checking a single frequency, but the actual performance is dependent on the individual MSs mobility. From the observations of the simulations, we determine the expected number of frequencies required to be checked for a certain proportion of scanning operations. For example, $x \%$ of the time, the MS can find a downlink channel with only scanning a single frequency. Results were obtained with a $95 \%$ level of confidence.

\section{Mobile Station Mobility Models}

For the simulations we used a variety of simulated and realworld mobility data. For the simulated mobility patterns we introduced two classes of user and two mobility models.

\section{User Classes}

1) Worker class - The worker mobility pattern models the scenario of a user that is commuting to and from work. The MS is given a set of random permanent home and work locations assigned within the area of WiMAX coverage. The worker travels from home to work and back once a day.

2) Wanderer class - The wanderer class is initially given a random start and destination location within the area of coverage. The wanderer moves towards its destination until it is reached. Then a new random destination is chosen and the wanderer continues moving towards this new destination. This repeats for the duration of the simulated day.

\section{Mobility Models}

The worker and wanderer classes are simulated using two different mobility models as shown in Figure 6. The first is the Manhattan model. The MS follows first the horizonal path, then the vertical path towards its destination (the reverse is true for the return course). The second is the direct model, where the MS follows a direct, straight-line, course across the area of coverage to its destination. Since the wanderer class continuously selects a new destination when reaches the previous one, it is following the random waypoint model [10].

In order to study the impact of slight variances in the repeated mobility patterns, we also implemented a possibility of detour for the worker class. With some probability, the worker class user will detour from its path by up to $2 \mathrm{~km}$. This was implemented for the Manhattan mobility model. 


\section{Real-World Mobility Traces}

In addition to the simulated mobility patterns, we also used real-world mobility data from the Automatic Packet Reporting System (APRS) project [2]. Since the locations for each MS were only updated on the order of every few minutes, the intermediate locations between updates are interpolated along a direct path in order to simulate the mobility second by second.

\section{Time-of-day Mobility Profiles}

In order to reduce the complexity of the simulation, a naive set of time-of-day mobility profiles was implemented. The day is divided into two time periods, morning and evening, with the goal of capturing a MSs regular commute to work in the morning in one time-of-day profile and its commute home in the second.

For the purpose of this work, we consider time-of-day parameters during the work week. The goal is to improve scanning times for users that typically repeat similar routes such as the daily commute to and from work. Therefore, we expect that the worker class will benefit the most from our strategies. We performed simulations for the WiMAX default, MFU and TOD frequency strategies in order to compare their results.

\section{E. Location Plus Trajectory Mobility Profiles}

In our evaluation of our LPT mobility profiles, we keep a total of four most probable lists for each of the $n \times m$ zones, as is depicted in Figure 3. We also let $n=m$ and tested two cases for the number of zones: 16 and 25 . We would expect that as you increase the number of zones, there should be an increase in performance since the MS can build a more finegrained mobility profile. We performed simulations for the WiMAX default, MFU and LPT frequency strategies in order to compare their results.

\section{Simulation Results}

The following sections show the simulation results of timeof-day mobility profiles with the frequency and previous handover strategies. We simulated the scanning operation when the MS is about to perform a handover. First we show the results for the simulated mobility patterns. Then we show the simulation results with the APRS mobility trace data.

\section{A. Time-of-Day Frequency Strategies}

In this section, we present the simulation results for scanning strategies using time-of-day mobility profiles. Figure 7 gives the results of the simulations for the worker class using the Manhattan mobility pattern. It shows the percentage of time that the MS succeeds in acquiring a channel with checking $1,2, \ldots, 20$ frequencies. We see significant improvement over our previous MFU strategy without the time-of-day mobility profiles.

The results are further summarized in Table II. MSs that used the time-of-day mobility profiles acquired the downlink

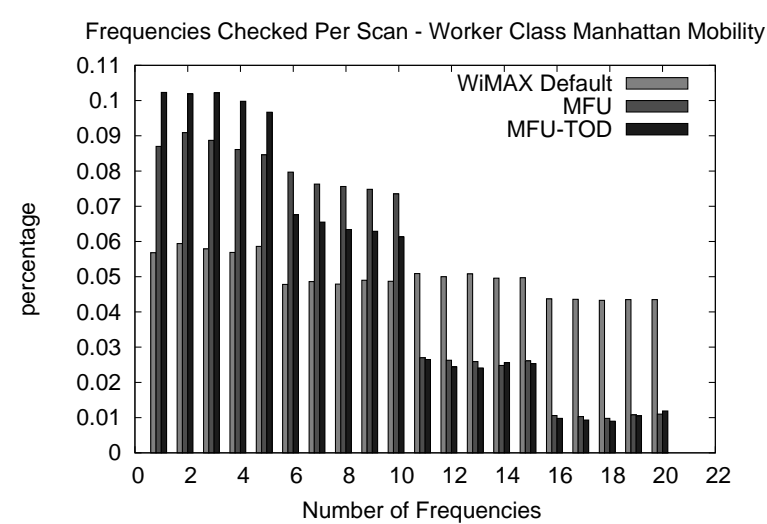

Fig. 7. Frequencies checked per scan - Worker class.

frequency $50.29 \%$ and $48.95 \%$ of the time with scanning only five frequencies for the Manhattan and Direct mobility models respectively. This is an increase over the strategy without time-of-day profiles which successfully acquired the frequency $43.73 \%$ of the time and the default WiMAX strategy which only succeeded $28.96 \%$ of the time with scanning only five frequencies.

\begin{tabular}{|l|c|c|}
\hline \multicolumn{3}{|c|}{ Scanning Results } \\
\hline & 5 Freq & 10 Freq \\
\hline Default & $28.96 \%(27.47 \%)$ & $53.16 \%(51.33 \%)$ \\
\hline MFU & $43.73 \%(39.92 \%)$ & $81.72 \%(74.57 \%)$ \\
\hline Time-of-Day & $50.29 \%(48.95 \%)$ & $82.36 \%(78.44 \%)$ \\
\hline LPT-16 & $73.23 \%(79.53 \%)$ & $97.17 \%(96.13 \%)$ \\
\hline LPT-25 & $78.71 \%(83.51 \%)$ & $97.47 \%(96.77 \%)$ \\
\hline
\end{tabular}

TABLE II

PROPORTION OF TIME SCANNING CAN BE COMPLETED WITH CHECKING 5, 10 FREQUENCIES - WORKER CLASS MANHATTAN (DIRECT) MOBILITY.

We then introduced the random detour to the worker class, Manhattan mobility, MSs daily commute. With a probability of $10 \%$, a detour would be taken either on the way to work or on the way home from work with equal probability. The detour could be up to $2 \mathrm{~km}$ in distance from the MS's path. We see that there is little impact on the performance of the strategy that uses time-of-day mobility profiles. The results are summarized in Table III.

\begin{tabular}{|l|c|c|}
\hline \multicolumn{3}{|c|}{ Scanning Results } \\
\hline & 5 Freq & 10 Freq \\
\hline Default & $28.87 \%$ & $53.09 \%$ \\
\hline MFU & $43.28 \%$ & $81.25 \%$ \\
\hline Time-of-Day & $49.85 \%$ & $81.64 \%$ \\
\hline
\end{tabular}

TABLE III

PROPORTION OF TIME SCANNING CAN BE COMPLETED WITH CHECKING 5, 10 FREQUENCIES - WORKER CLASS MANHATTAN MOBILITY WITH DETOUR.

Finally, since not all users may follow a predictable mobility pattern, we ran a simulation with the wanderer class of MS. As 
expected, since the wanderer class has no predictable mobility pattern, there was no improvement, over the original MFU strategy, in the number of frequencies checked during the scanning operation.

\section{B. Location Plus Trajectory Frequency Strategies}

In this section, we present the simulation results for scanning strategies using time-of-day mobility profiles. Figure 8 gives the results of the simulations for the worker class using the Manhattan mobility pattern. It shows the percentage of time that the MS succeeds in acquiring a channel with checking $1,2, \ldots, 20$ frequencies. We see a significant improvement over the previous MFU strategy and the TOD mobility profiles.

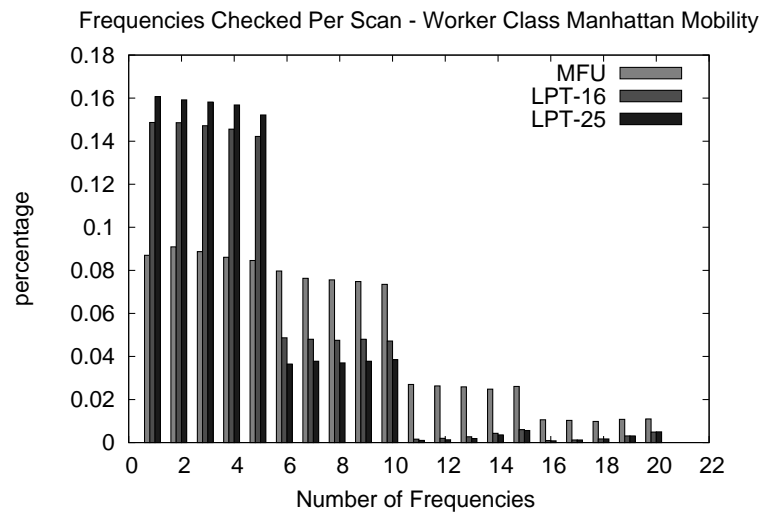

Fig. 8. Frequencies checked per scan - Worker class.

The results for the worker class mobility are further summarized in Table II. We observe that MSs using the LPT-16 and LPT-25 profiles acquired the downlink frequency $73.23 \%$ and $78.71 \%$ of the time with scanning only five frequencies. This compares to $28.96 \%$ for WiMAX default, $43.73 \%$ for the original MFU strategy and $50.29 \%$ for the TOD mobility profile.

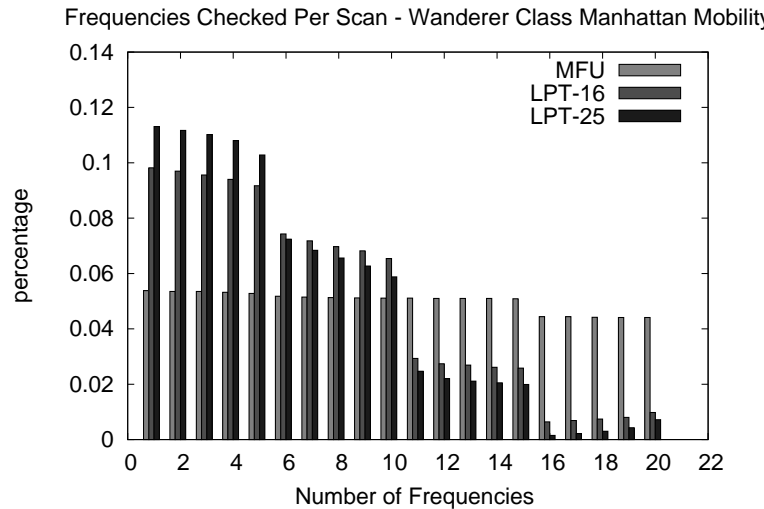

Fig. 9. Frequencies checked per scan - Wanderer class - LPT mobility profiles.
Finally, as in the previous section, since not all users may follow a predictable mobility pattern, we simulated the wanderer class. Figure 9 shows the performance of our LPT strategies with the wanderer class using the Manhattan mobility pattern. In the case of the wanderer class, we do see improvement of using the LPT-16 and LPT-25 mobility profiles over the original MFU strategy. The LPT-16 and LPT-25 strategies acquired the downlink frequency $47.65 \%$ and $54.58 \%$ of the time respectively with scanning only five frequencies. This is an increase over the original MFU strategy which successfully acquired the frequency $26.68 \%$ of the time within five frequencies. The results for the wanderer class mobility are summarized in Table IV.

It is worth noting that as expected, the LPT- 25 mobility profile performed better when compared to the LPT-16 mobility profile. With an increased number of zones, each zone covered a smaller area thereby giving the MS a finer grained profile.

\begin{tabular}{|l|c|c|}
\hline \multicolumn{3}{|c|}{ Scanning Results } \\
\hline & 5 Freq & 10 Freq \\
\hline MFU & $26.68 \%$ & $52.37 \%$ \\
\hline LPT-16 & $47.65 \%$ & $82.59 \%$ \\
\hline LPT-25 & $54.58 \%$ & $87.37 \%$ \\
\hline
\end{tabular}

TABLE IV

PROPORTION OF TIME SCANNING CAN BE COMPLETED WITH CHECKING 5 , 10 FREQUENCIES - WANDERER CLASS MANHATTAN MOBILITY.

\section{Time-of-Day Previous Handover Strategy}

In this section, we present the simulation results for the previous handover strategy when using time-of-day mobility profiles. Recall that the previous handover strategy enables the MS using optional neighbor advertisement messages, sent by its currently serving BS, along with a mobility profile to make better choices during scanning. We used the same time-of-day mobility profiles as in the frequency strategies. In this simulation scenario, the WiMAX default was to simply scan the neighbors in the order given by the MOB_NBR-ADV messages. Figure 10 shows the results of the simulations for the worker class using the Manhattan mobility pattern. We see a significant improvement over the previous strategy without the time-of-day mobility profiles.

The results are further shown in Table $\mathrm{V}$ where we see dramatic improvements in target BS selection when using time-of-day mobility profiles. This is intuitive since a worker class MS following a return path likely traverses the set of $\mathrm{BSs}$ in reverse order

\begin{tabular}{|l|c|c|}
\hline \multicolumn{3}{|c|}{ Previous Handover Results } \\
\hline & Manhattan & Direct \\
\hline Default & $19.26 \%$ & $18.48 \%$ \\
\hline MFU & $36.29 \%$ & $40.11 \%$ \\
\hline Time-of-Day & $95.54 \%$ & $99.19 \%$ \\
\hline
\end{tabular}

TABLE V

PROPORTION OF TIME HANDOVER TARGET BASE STATION CHOSEN ON FIRST ATTEMPT - MANHATTAN/DiRECT. 


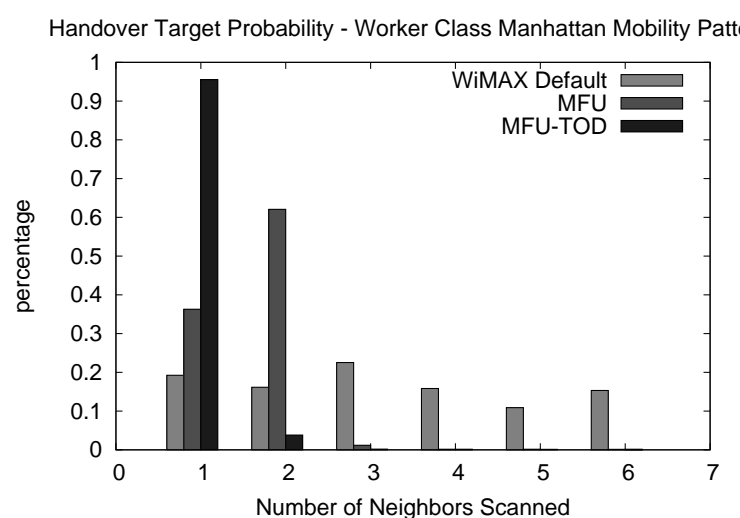

Fig. 10. Base station neighbor scans per handover - Worker class.

As in the previous section, we also studied the effect of a detour on the worker class MSs using the Manhattan mobility model. As before, with a $10 \%$ probability, the worker MS would make a detour of up to $2 \mathrm{~km}$ away from its path during either its route to work, or its route home. The results show that there is minimal impact on the performance over the nondetour scenario.

Finally, as with our frequency strategies, we simulated the wanderer class and expected to see little advantage to building user mobility profiles for this class of user. We also simulated a mixture of worker and wanderers. As expected, since the wanderer class has no predictable mobility pattern, there is no improvement in the scanning operation when selecting the target BS. With a mixture of Wanderer (20\%) and Workers $(80 \%)$ we still see significant overall improvement. The results are summarized in Table VI.

\begin{tabular}{|l|c|}
\hline \multicolumn{2}{|c|}{ Previous } \\
\hline & Mandover Results \\
\hline Default & $18.34 \%$ \\
\hline MFU & $34.49 \%$ \\
\hline Time-of-Day & $82.43 \%$ \\
\hline \multicolumn{2}{|c|}{ TABLE VI }
\end{tabular}

PROPORTION OF TIME HANDOVER TARGET BASE STATION CHOSEN ON FIRST ATTEMPT - MIXED CLASSES (80\%/20\%), MANHATTAN MOBILITY.

\section{Mobility Trace Data Simulation}

We present the results using mobility trace data from the APRS project. We performed a simulation with 35 MS mobility traces and our two time-of-day and location plus trajectory mobility profiles.

In Figure 11, we present the results for the previous handover strategy using the time-of-day mobility profiles. Here we see an improvement of handover scanning frequency selection. When using time-of-day mobility profiles, the MS successfully acquires the correct BS target on the first attempt $63.25 \%$ of the time versus $54.19 \%$ for our original MFU strategy and only $17.64 \%$ for the WiMAX default strategy.

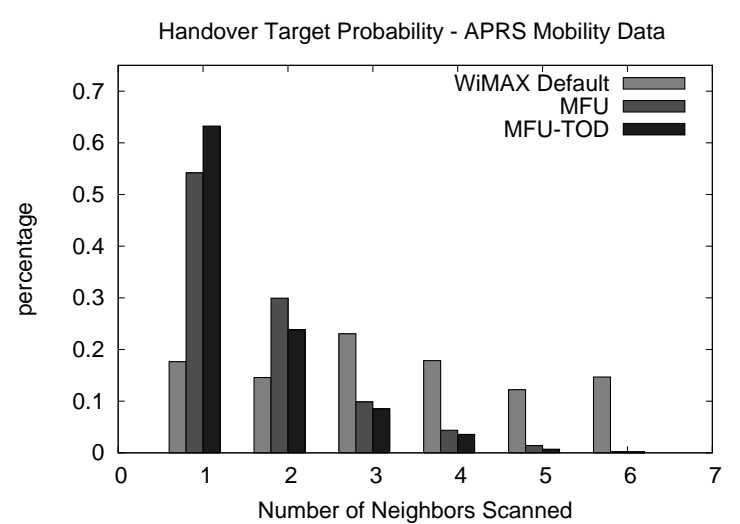

Fig. 11. Base station neighbor scans per handover - APRS mobility data.

Figure 12 shows the results of our frequency scanning strategies for LPT with 16 and 25 zones against our original MFU strategy using the APRS mobility trace data. We observe significant improvements through the use of the LPT mobility profiles.

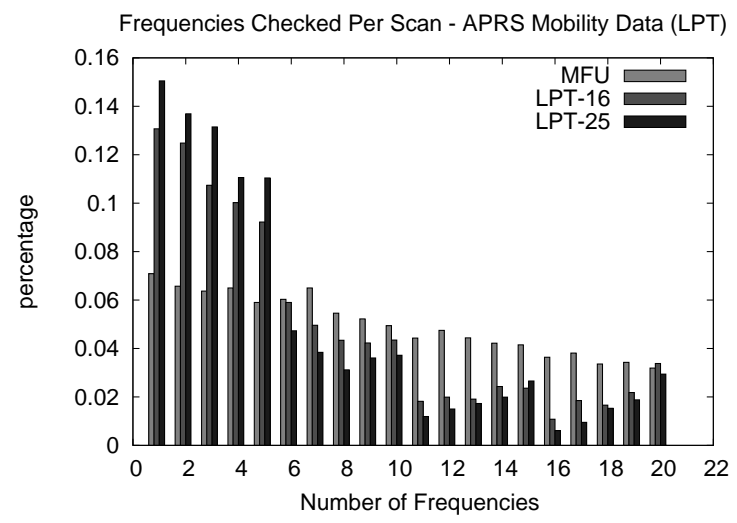

Fig. 12. Frequencies checked per scan - APRS mobility data, LPT mobility profiles.

The results are further summarized in Table VII. MSs that used the LPT-16 and LPT-25 mobility profiles acquired the downlink frequency $55.53 \%$ and $63.99 \%$ of the time with scanning only five frequencies. This is an increase over the MFU strategy which successfully acquired the frequency $32.43 \%$ of the time and the default WiMAX strategy which only succeeded $23.36 \%$ of the time with scanning only five frequencies.

\section{CONCLUSiOn AND Future Work}

In our work, we have focused on improving the scanning times for WiMAX/802.16 MSs during handovers. We have introduced two MS based mobility profiles, time-of-day and location plus trajectory, using our MFU scanning strategy, to aid in reducing the number of frequencies checked while 


\begin{tabular}{|l|c|c|}
\hline \multicolumn{3}{|c|}{ Scanning Results } \\
\hline & 5 Freq & 10 Freq \\
\hline Default & $23.36 \%$ & $46.53 \%$ \\
\hline MFU & $32.43 \%$ & $60.58 \%$ \\
\hline LPT 16 Zones & $55.53 \%$ & $79.31 \%$ \\
\hline LPT 25 Zones & $63.99 \%$ & $83.01 \%$ \\
\hline
\end{tabular}

TABLE VII

PROPORTION OF TIME HANDOVER SCANNING CAN BE COMPLETED WITH CHECKING 5, 10 FREQUENCIES - APRS MOBILITY DATA.

scanning to find a downlink from a BS. We have provided performance results from a set of experiments based on simulated and real-world mobility traces. Our mobility profiles are MS based and require no additional network support.

Through the simulated mobility experiments, we have shown that using either TOD or LPT mobility profiles improves handover scanning performance over that of the WiMAX/802.16 default and our previous work. For example, the worker class using the Manhattan mobility model could complete scanning within five frequencies on average $50.29 \%$, $73.23 \%$ and $78.71 \%$ of the time for the TOD, LPT- 16 and LPT25 mobility profiles respectively. This is an increase over the $43.73 \%$ seen from our original MFU strategy and the $28.96 \%$ for the WiMAX default. We see similar improvements when using the Direct mobility model. Additionally, the LPT mobility profile provided significant improvement for the wanderer mobility class where the TOD profile did not. We observed MSs completing scanning within five frequencies $47.65 \%$ and $54.58 \%$ of the time on average for LPT-16 and LPT-25 as compared to the $26.68 \%$ seen for our original MFU strategy.

Using the real-world APRS mobility data, along with the LPT mobility profiles, the MS can complete scanning within five frequencies $55.53 \%$ and $63.99 \%$ of the time for LPT16 and LPT-25 respectively. This is an improvement over the $32.43 \%$ for our original MFU strategy and the $23.36 \%$ for the WiMAX default strategy. We also show improvement in scanning performance when using the TOD mobility profile for the previous handover strategy when simulating with the APRS mobility trace data. However, a more sophisticated set of TOD mobility profiles should be implemented. In addition, other sets of mobility data should be examined, such as users of cellular telephones.

For the time-of-day mobility profiles, the MS is maintaining information on windows of times that it a) uses a certain frequency, and b) performs a handover from one BS to another. This window of time is not specific to any particular date, just a particular day or category of day of the week (i.e. Monday-Friday, Weekend). The profiles built with the initial frequency strategy will be of little other use since it only relates frequency use to a time period. With profiles for the handover strategies, we can extrapolate a general pattern of BSs traversed (i.e. user often travels through BS 1, BS 2, then BS 3 on Weekday mornings). This should not be a security or privacy issue since the information maintained in the profiles is rather coarse grained, is only maintained within the MS's internal memory and not shared with any other entity.

Future work includes providing a larger set of finer grained time-of-day mobility profiles. There is the additional problem of developing a MS algorithm for building its optimal set of time-of-day profiles. The set of time periods that need to be profiled depends on the mobility patterns of individual MSs.

A further extension to the LPT model is to include building profiles for different routes a MS may take. The MS could then determine which route it is currently following and use this on which to base its scanning decisions. Additionally, the assignment of LPT zones to the coverage area could be done differently. Instead of simply dividing the coverage area into a grid, the MS could examine the various routes it takes and determine the pattern of roads traversed or multiple zones containing similar frequency use could be merged into superzones.

\section{ACKNOWLEDGEMENTS}

Research supported in part by the Natural Sciences and Engineering Research Council of Canada (NSERC) and Mathematics of Information Technology and Complex Systems (MITACS).

\section{REFERENCES}

[1] IEEE 802.16-2008, "IEEE Standard for Local and Metropolitan Area Networks - Part 16: Air Interface for Fixed Broadband Wireless Access Systems," 2008.

[2] Automatic Packet Reporting System, http://www.aprs.org and http://www.findu.com/

[3] D. Agrawal and Q.-A. Zeng. Introduction to Wireless and Mobile Systems. Thomson-Engineering, first edition, 2002.

[4] M. Aguado, E. Jacob, P. Saiz, J. Matias, M. Higuero, N. Toledo and M. Berbineau, "Scanning on handover enhancement issues in video application deployments on WiMAX Mobile Networks," IEEE International Symposium on Broadband Multimedia Systems and Broadcasting, pp. 1-7, 2008.

[5] Z. Becvar, P. Mach and R. Bestak, "Optimization of handover scanning procedure in WiMAX networks with relay stations," 3rd International Symposium on Wireless Pervasive Computing, pp. 581-585, 2008

[6] P. Boone, M. Barbeau, and E. Kranakis, "Strategies for Fast Scanning, Ranging and Handovers in WiMAX/802.16," International Journal of Communication Networks and Distributed Systems, Vol. 1, No.4/5/6, pp. 414 - 432, 2008. (Also in proceedings of 2nd International Conference on Networks and Workshops, AccessNets 2007)

[7] S. Choi, G. Hwang, T. Kwon, A. Lim, and D. Cho., "Fast handover scheme for Real-Time Downlink Services in IEEE 802.16e BWA System," In Vehicular Technology Conference, 2005. VTC 2005-Spring. pp. 2028-2032, January 2005.

[8] ETSI, Universal Mobile Telecommunication System (UMTS), "Selection procedures for the choice of radio transmission technologies of the UMTS," UMTS 30.03 Version 3.2.0, 1998-04. Available at http://www.3gpp.org/ftp/Specs/html-info/3003U.htm

[9] F.-Y. Hsieh, Y.-W. Chen, and P-W.Wu. "Cross Layer Design of Handoffs in IEEE 802.16e Networks", International Computer Symposium, ICS 2006, pages 703-708, 2006.

[10] D. B. Johnson and D. A. Maltz, "Dynamic source routing in ad hoc wireless networks," Mobile Computing, 153-181, 1996.

[11] K. Kim, C. Kim, T. Kim, "A Seamless Handover Mechanism for IEEE 802.16e Broadband Wireless Access," Proceedings of International Conference of Computational Science (ICCS) 2005.

[12] R. Rouil and N. Golmie, "Adaptive Channel Scanning for IEEE 802.16e," Proceedings of 25th Annual Military Communications Conference (MILCOM 2006), Washington, D.C., October pp. 23-25, 2006.

[13] P. van de Berg, "Carrier Scanning in Cellular Networks," Patent number: WO/2000/078075, World Intellectual Property Organization, http://www.wipo.int, 2000 